\title{
Integrated Radionuclide Release: Tests and Model Development (Integrated Testing)
}

\author{
Brian Viani
}

August 31, 1995

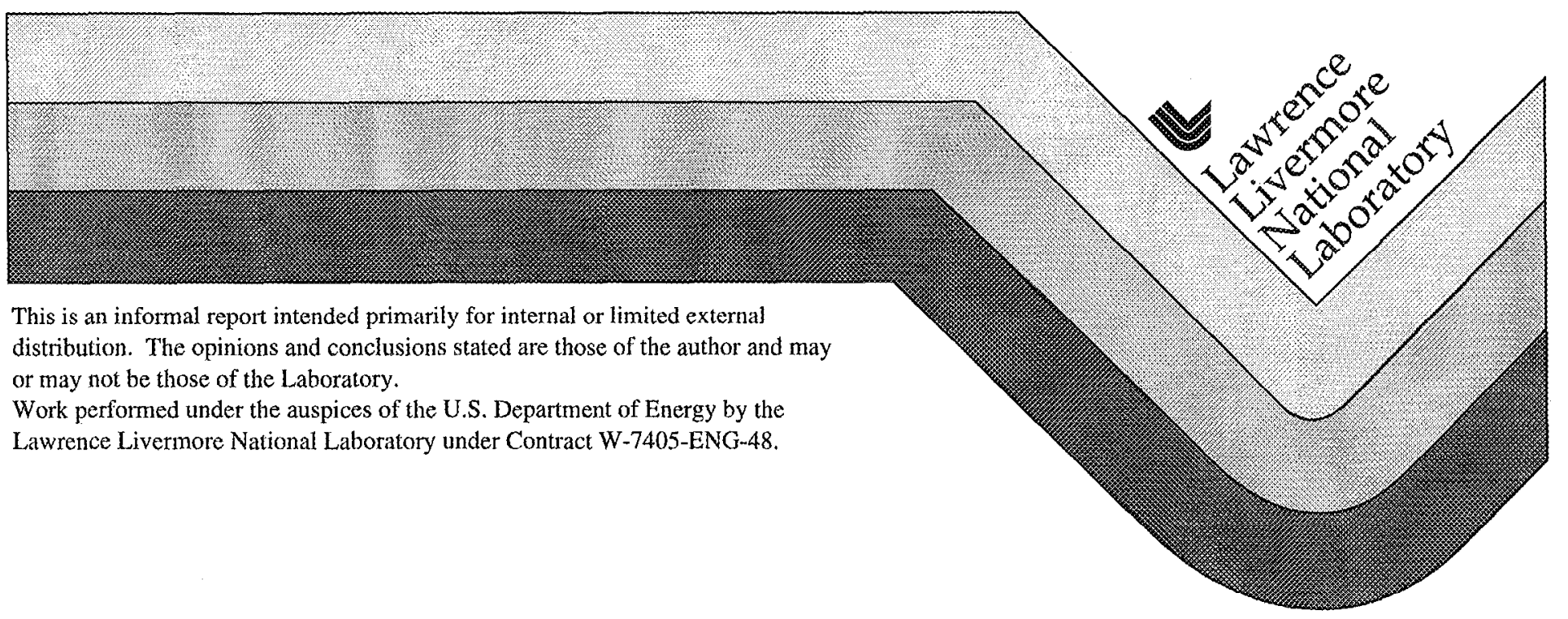




\section{DISCLAIMER}

This document was prepared as an account of work sponsored by an agency of the United States Government. Neither the United States Government nor the University of California nor any of their employees, makes any warranty, express or implied, or assumes any legal liability or responsibility for the accuracy, completeness, or usefulness of any information, apparatus, product, or process disclosed, or represents that its use would not infringe privately owned rights. Reference herein to any specific commercial product, process, or service by trade name, trademark, manufacturer, or otherwise, does not necessarily constitute or imply its endorsement, recommendation, or favoring by the United States Government or the University of California. The views and opinions of authors expressed herein do not necessarily state or reflect those of the United States Government or the University of California, and shall not be used for advertising or product endorsement purposes.

This report has been reproduced

directly from the best available copy.

Available to DOE and DOE contractors from the

Office of Scientific and Technical Information

P.O. Box 62, Oak Ridge, TN 37831

Prices available from (423) 576-8401

Available to the public from the

National Technical Information Service

U.S. Department of Commerce

5285 Port Royal Rd.,

Springfield, VA 22161 
University of California

IL Lawrence Livermore

National Laboratory

YUCCA MOUNTAIN PROJECT

Scientific Investigation Plan

Subject:

Integrated Radionuclide Release: Tests and Model

Development (Integrated Testing)
No.: $\quad$ SIP-IT-01

Revision: 0

Change Notice: N/A

Page: 1 of 31

Training Aequired: Yes $\square$ No

Comments:

N/A

AUTHOR:

Brian Viani

REVISION HISTORY

Rev. No. CNNo. Effective Date Déscription of Revision/CN

0

08/31/95 Original issue.

APPROVALS: 
Scientific Investigation Plan

for

YMP WBS Element 1.2.3.10.3.1

Integrated Radionuclide Release:

Tests and Model Development

(Integrated Testing)

(Supersedes SIP-4)

Lawrence Livermore National Laboratory
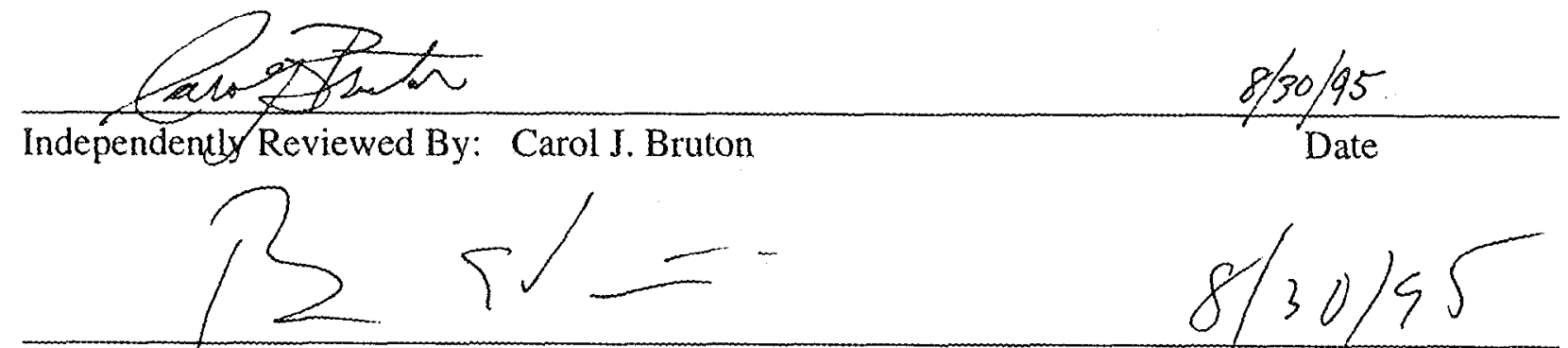

Prepared By: Brian E. Viani

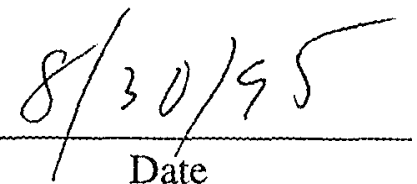




\section{Table of Contents}

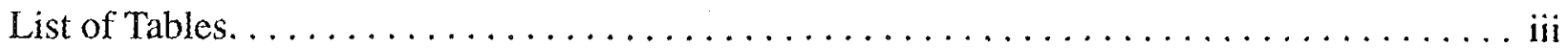

List of Figures. . . . . . . . . . . . . . . . . . . . .

Preface. . . . . . . . . . . . . . . . . . . . . . . . . . . . .

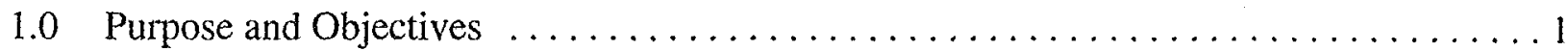

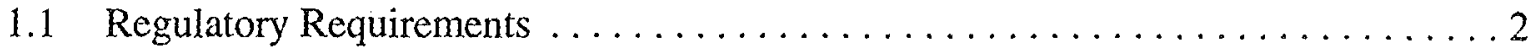

1.2 Relationship between this SIP and the SCP $\ldots \ldots \ldots \ldots \ldots \ldots \ldots \ldots$

1.3 Interfaces and Information Flow $\ldots \ldots \ldots \ldots \ldots \ldots \ldots \ldots \ldots \ldots \ldots$

1.3.1 Activities That Define the Near Field Environment and Models of Chemical, Mineralogical, and Hydrological Evolution in the Post Emplacement

Period . . . . . . . . . . . . . . . . . . . . . . . 6

1.3.1.1 Interface with Groundwater Chemistry Model Activities

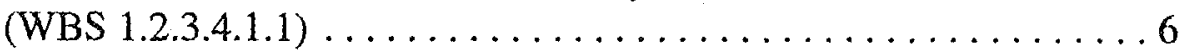

1.3.1.2 Interface with NFE Geochemistry Activities (WBS 1.2.3.12.1) _ . 6

1.3.1.3 Interface with Altered Zone activities (WBS 1.2.3.10) . . . . . . 6

1.3.1.4 Interface with Hydrological Properties of the NFE

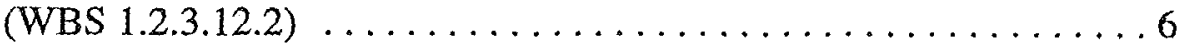

1.3.2 Activities That Define Material Properties of Flow-Path Components and

Fluids . . . . . . . . . . . . . . . . . . . . . . . 6

1.3.2.1 Interface with Waste Form Testing (WBS 1.2.2.4) . . . . . . . .6

1.3.2.2 Interface with Waste Package Materials activities (WBS 1.2.2.5) . . 7

1.3.2.3 Interface with NFE Man Made Materials activities

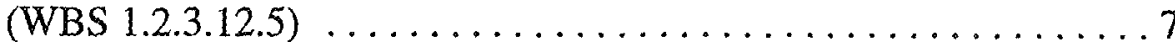

1.3.3 Activities Supplying Supporting Data for Radionuclide Sorption, Transport, and Speciation Modeling $\ldots \ldots \ldots \ldots \ldots \ldots \ldots \ldots \ldots \ldots$

1.3.3.1 Interface with Dynamic Transport, Sorption and Diffusion Activities

(WBS 1.2.3.4.1.4.1/2 \& 1.2.3.4.1.2.1/3) . . . . . . . . . . 8

1.3.3.2 Interface with EQ3/6/GEMBOCHS Activities (WBS 1.2.3.4.2.1/2) . 8

1.3.3.3 Interface with Thermodynamic Data Activities (WBS 1.2.3.10.3.2

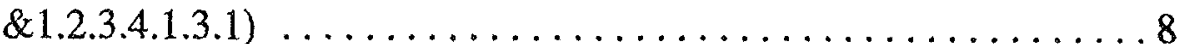

1.3.4 Interface with EBS/NFE Subsystem Performance Assessment . . . . . . 8

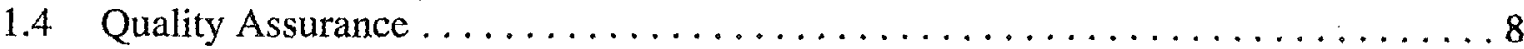

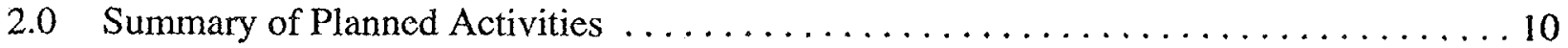

2.1 Experimental Measurement and Compilation of Model Parameters ... . . . . 10

2.1.1 Planning/data assessment (Activity $\mathrm{G}-20-8) \ldots \ldots \ldots \ldots \ldots \ldots \ldots \ldots$

2.1.2 Transport model parameter experiments necessary to define the source term

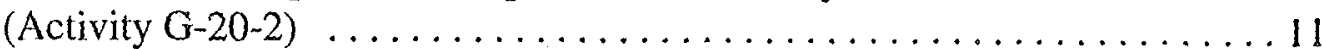

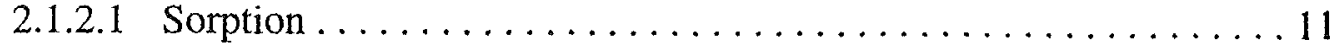

2.1.2.2 Diffusion . . . . . . . . . . . . . . . . . . . . .

2.2 Experiments to Measure the Transport of Radionuclides $\ldots \ldots \ldots \ldots \ldots \ldots \ldots$ 
2.2.1 Planning/data assessment (Activity G-20-8) ..............

2.2.2 Radionuclide transport experiments necessary to define the source term

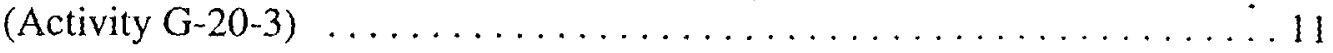

2.2.3 Interactions of materials at repository conditions (Activity G-20-5) $\ldots \ldots 12$

2.3 The Application, Development, and Validation of Models . . . . . . . . 12

2.3.1 Source term prediction and model development (Activity G-20-6) . . . . 12

2.3.2 Model validation (Activity G-20-7) $\ldots \ldots \ldots \ldots \ldots \ldots \ldots \ldots \ldots \ldots$

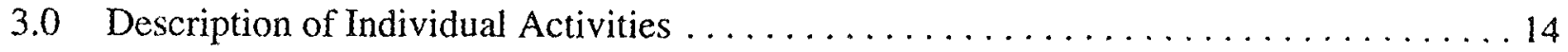

3.1 Transport Model Parameter Experiments Necessary to Define the Source Term

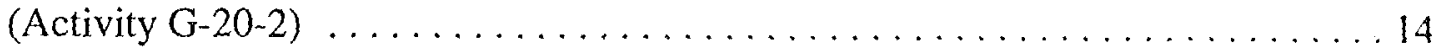

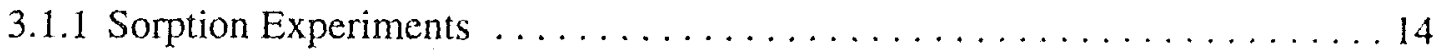

3.1.2 Diffusion experiments ........................... 15

3.2 Radionuclide Transport Experiments Necessary to Define the Source Term

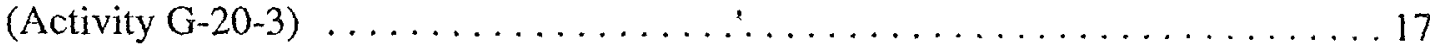

3.3 Interaction of Materials at Repository Conditions (Activity G-20-5) $\ldots \ldots \ldots 18$

3.4 Source Term Prediction and Model Development (Activity G-20-6) . . . . . 19

3.5 Source Term Model Validation (Activity G-20-7) . . . . . . . . . . . . . 20

3.6 Data Assessment/Planning (Activity G-20-8) $\ldots \ldots \ldots \ldots \ldots \ldots \ldots \ldots \ldots$

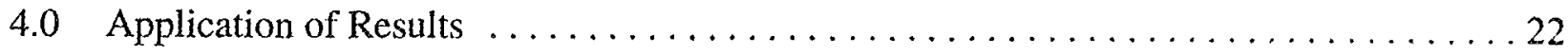

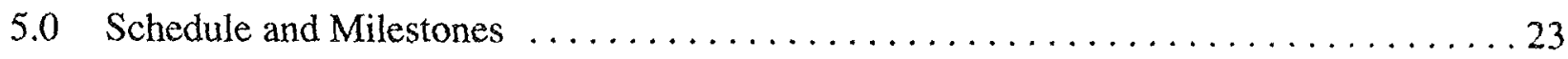

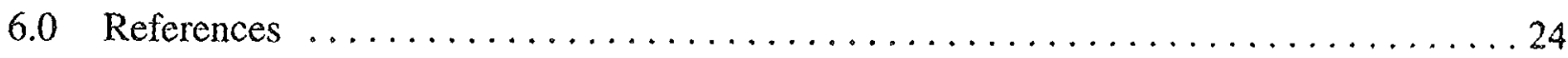




\section{List of Tables}

Table 1. Integrated Testing activities grouped by SCP study $\ldots \ldots \ldots \ldots \ldots \ldots$

Table 2. Activities for Integrated Testing ......................... 10

Table 3. Schedule for Integrated Testing activities..................... 23 


\section{List of Figures}

Figure 1. Schematic representation of a waste package and the EBS/NFE showing flow-path components through which radionuclides may travel: (1) breach in waste package and associated container filler materials and corrosion products, (2) backfill/packing material, (3) fractured concrete, (4) fibercrete drift covering, (5) base rock, (6) altered Topopah Spring iuff, and (8) unaltered

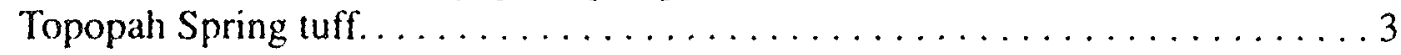

Figure 2. Information flow and relationship of Integrated Testing to other tasks.......5 


\section{PREFACE}

The Department of Energy's Yucca Mountain Site Characterization Project (YMP) is evaluating a site at Yucca Mountain, Nevada as a potential repository for the disposal of high-level nuclear waste. Credible bounding estimates of the type, concentration, and nature of the radionuclides that might potentially escape the engineered barrier system/near field environment (EBS/NFE) (i.e., the source term) are necessary prerequisites to assessing the expected performance of a potential repository. Estimating the source term will require a combination of experimental and modeling tasks that are designed to assess the release of radionclides from the waste form and their transport through and interaction with the components of the EBS/NFE.

The Integrated Radionuclide Release: Tests and Model Development activity (Integrated Testing) at Lawrence Livermore National Laboratory (LLNL) is an experimental and modeling activity that is specifically concerned with radionuclide transport through and interaction with the components of the EBS/NFE. As such, this activity is the link between waste-form degradation activities and far-field transport activities. Performance analysis of the EBS/NFE subsystem is an important component of the total system performance analyses (TSPA) that will be used to evaluate repository performance. 
The role of the Integrated Radionuclide Release: Tests and Model Development activity (Integrated Testing) is to bound the flux of radionuclides that pass the Engineered Barrier System/Near Field Environment (EBS/NFE). The purpose of the work outlined in this Scientific Investigation Plan (SIP) is to measure and model the potential for transport of radionuclide elements from the wastc form through the introduced matcrials, their alteration products, and altered host rock that will comprise the post-emplacement near field environment of the potential repository at Yucca Mountain. Experimental measurements will be combined with conceptual and mechanistic models to bound the concentrations of radionuclide elements that will be released from the EBS/NFE. The estimated releases will be used by the EBS/NFE Subsystem Performance Assessment task (EBS/NFE PA) to calculate the source term to be used in Total System Performance Assessment (TSPA).

The path a radionuclide may take from the waste form through the EBS/NFE to the unaltered repository rock may intersect one or more of the following materials (hereafter referred to as flowpath components):

- Waste package filler and packing materials and their degradation products

- Waste package metallic corrosion resistant barrier

- Waste package metallic corrosion allowance barrier and its corrosion products

- Waste package non-metallic barrier and its alteration products

- Backfill and packing (e.g. crushed tuff) and their alteration products

- Inver materials (e.g., concrete and crushed tuff) and their degradation products

- Topopah Spring tuff (host rock) that has been altered by hydrothermal interaction with ambient fluids and via interaction with EBS/NFE components.

The physical and chemical properties of these materials as initially introduced to the repository are expected to undergo alteration to various degrees during the period of substantially complete containment. Thus, it is the alteration products of EBS/NFE components that will define the chemical and physical environment through which radionuclides released from the waste form must traverse.

The physical state of potential flow-path components may range from unconsolidated fine-grained materials such as corrosion products derived from the waste package corrosion allowance barrier, to consolidated and fractured materials such as altered Topopah Spring tuff and concrete. Nongaseous radionuclide elements in the form of dissolved or colloidal species will move in an aqueous phase through the EBS/NFE via diffusive and advective/dispersive transport. The movement of the radionuclide-bearing aqueous phase will be strongly affected by the properties of the materials contacted along the flow path and their liquid saturation state.

The sorptive capacity and chemical properties of potential EBS/NFE components will vary considerably, and may include strongly sorbing materials such as Fe-oxide corrosion products, hyperalkaline materials such as concrete and fibercrete, and material with properties similar to the host rock, such as crushed tuff backfill and/or invert. 
It is anticipated (1) that the hydrologic regime in the EBS/NFE will remain predominantly unsaturated during the period of substantially complete containment and during the period when radionuclides are released from the waste form. Although the EBS/NFE as a whole may be unsaturated, episodic flow of water through focused or preferential flow paths cannot be ruled out (1). Hence, transport of radionuclides through the EBS/NFE could range from transport through unsaturated unconsolidated porous media (e.g., backfill, corrosion products), to transport along saturated or near-saturated preferential flow paths (e.g., fractured concrete, altered tuff). It is anticipated that the temperature regime over which transport of radionuclides is likely to occur in the EBS/NFE will range from near the nominal boiling point of in-situ fluids to near ambient; i.e., radionuclide transport through the EBS/NFE is expected to occur after the initial thermal pulse has passed and temperatures are near boiling and below.

\subsection{Regulatory Requirements}

The information developed under this SIP will be used to assess the compliance with the performance objective for the EBS to control the rate of release of radionuclides (10 CFR 60.113). The information generated from this plan will serve as a component (along with information generated in the plans for waste form and waste package testing activities and by EBS/NFE PA) in determining the source term for TSPA modeling for use in showing compliance with the Environmental Protection Agency requirements contained in 40 CFR 191.13. The information gathered will also contribute toward estimating the source term in the calculation of long term cumulative releases (10 CFR 960.3-1-5).

\subsection{Relationship between this SIP and the SCP}

The Integrated Testing SIP addresses the following issues and information needs (IN) that are described in the 1988 Statutory Draft of the YMP Site Characterization Plan (SCP) (9).

Issue 1.5: Will the waste package and repository engineered barriers meet the performance objective for radionuclide release as required by 10 CFR 60.113 ?

IN 1.5.3 Scenarios and models needed to predict the rate of radionuclide release from the waste package and engineered barrier system.

IN 1.5.5 Determination of the amount of radionuclides leaving the near-field environment of the waste package.

Through input to the above issues and information needs, the results of the Integrated Testing activities will provide data to help resolve Issues 1.1, 1.5, and 1.9.

The scope of this SIP is broadly defined by the information needs listed above and the discussions in the SCP (Chapter 8, Section 8.3.5.10, Engineered Barrier System Release Rates) of the studies to be undertaken to provide information to resolve Issue 1.5. The purpose of the Integrated Testing activity as defined in the original SIP (10) and in the SCP was to develop a source term model 
for radionuclide transport in the NFE. At the time the SCP and the original version of this SIP were written, the reference waste package/EBS design did not include materials other than the waste package and the altered host rock. Hence, these documents did not discuss the effect of other materials, such as backfill, packing, invert, etc. on the transport of radionuclides through the NFE. Although the scope of this SIP is broadened to account for these materials, the overall objective remains the same; to evaluate the interactions between EBS/NFE components as they affect the transport of radionuclides so as to bound the radionuclide source term to be used in EBS/ NFE PA calculations. A schematic representation of the EBS/NFE and flow-path components for which radionuclide transport measurements and models are necded is shown in Figure 1. The ac-

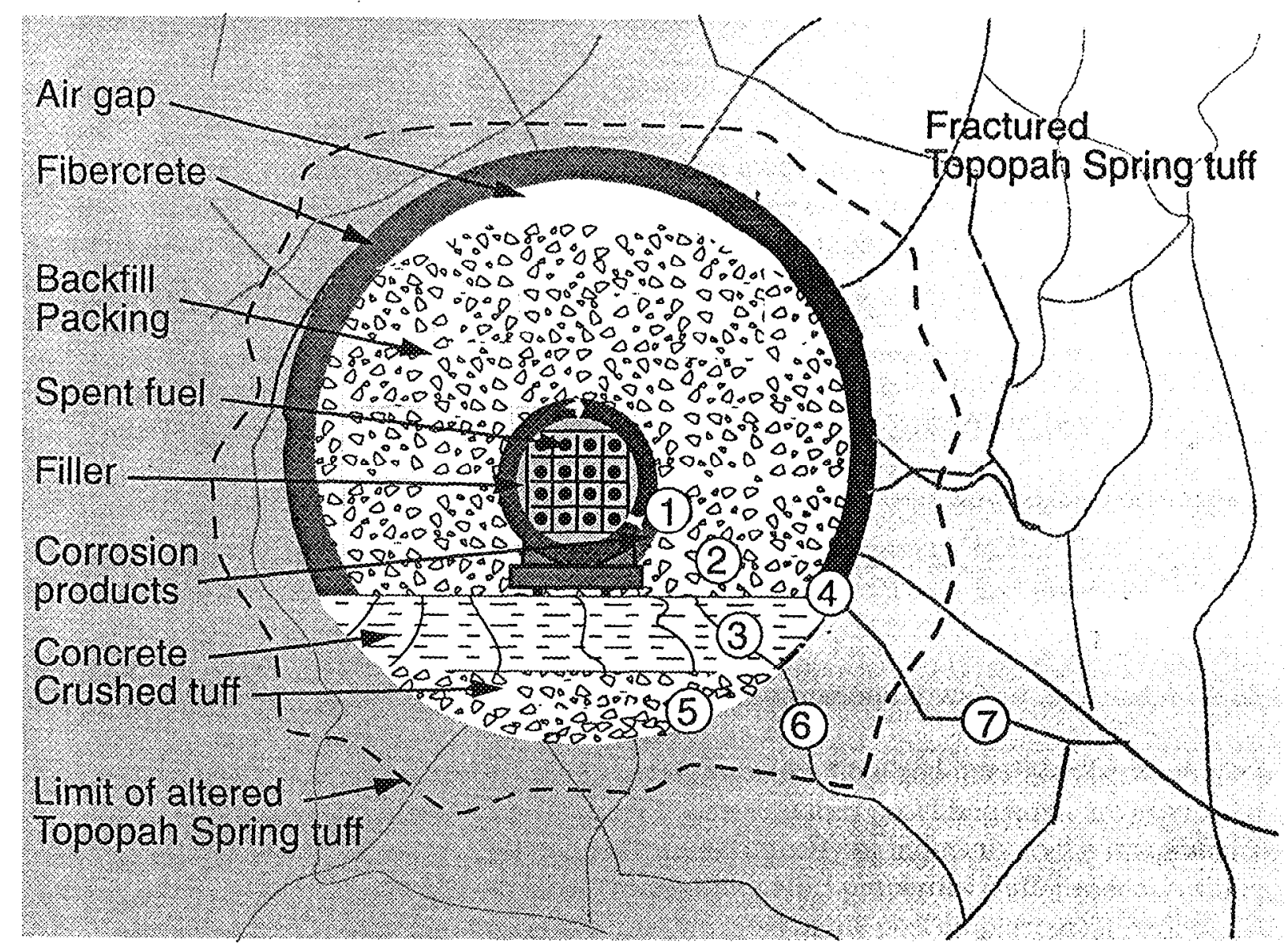

Figure 1. Schematic representation of a waste package and the EBS/NFE showing flow-path components through which radionuclides may travel: (1) breach in waste package and associated container filler materials and corrosion products, (2) backfill/packing material, (3) fractured concrete, (4) fibcrcrete drift covering, (5) base rock, (6) altered Topopah Spring tuff, and $(8)$ unaltered Topopah Spring tuff.

tivities comprising the Integrated Testing task may be broadly related to the SCP according to Table 1 . 
Table 1. Integrated Testing activities grouped by SCP study

\begin{tabular}{|c|c|}
\hline SCP Study & Integrated Testing Activity \\
\hline $\begin{array}{l}\text { Determine radionuclide transport } \\
\text { parameters (SCP IN 1.5.5, Investiga- } \\
\text { tion 1.5.5.1, Activities 1.5.5.1.1 and } \\
\text { 1.5.5.1.2) }\end{array}$ & $\begin{array}{l}\text { Transport model parameter experiments necessary to } \\
\text { define the source term (Activity G-20-2) } \\
\text { Radionuclide transport experiments necessary to define } \\
\text { the source term (Activity G-20-3) } \\
\text { Interactions of materials at repository conditions } \\
\text { (Activity G-20-5) } \\
\text { Planning/data assessment (Activity G-20-8) }\end{array}$ \\
\hline $\begin{array}{l}\text { Radionuclide transport modeling in } \\
\text { the near-field waste package environ- } \\
\text { ment (SCP IN 1.5.5, Investigation } \\
\text { 1.5.5.2, Activities 1.5.5.2.1 and } \\
\text { 1.5.5.2.2) }\end{array}$ & $\begin{array}{l}\text { Source term prediction and model development } \\
\text { (Activity G-20-6) } \\
\text { Model validation (Activity G-20-7) }\end{array}$ \\
\hline $\begin{array}{l}\text { Waste package performance assess- } \\
\text { ment model development (SCP IN } \\
\text { 1.5.3, Investigation } 1.5 .3 .5 \text {, Activity } \\
\text { 1.5.3.5.3) }\end{array}$ & $\begin{array}{l}\text { Interactions of materials at repository conditions } \\
\text { (Activity G-20-5) } \\
\text { Source term prediction and model development } \\
\text { (Activity G-20-6) }\end{array}$ \\
\hline
\end{tabular}

\subsection{Interfaces and Information Flow}

Figure 2 shows the information flow and interfaces between the Integrated Testing task and other EBS/NFE activities. The activities providing input are grouped into three categories based on the information they provide directly or indirectly to the Integrated Testing task: 1) activities that provide information regarding NFE properties and/or models of mineralogical, chemical, and hydrological evolution of the NFE in the post emplacement period; 2) activitics that provide information regarding material properties of radionuclide-bearing solutions, flow-path components and fluid phases; and 3) activities that provide data and parameters for radionuclide sorption, diffusion, transport and speciation. These groupings are approximate and some of the activities fall into more than one group. 
NFE and Models of Mineralogical and Chemical Evolution
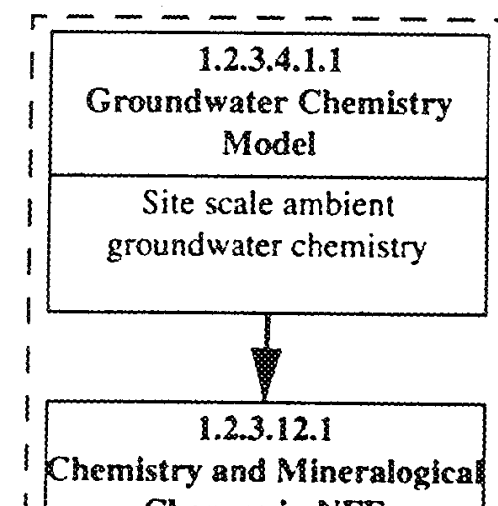

1 Changes in $N F E$

1 Evolution of posi-emplacement

1 water chemistry and

1 mineralogy in the NFE
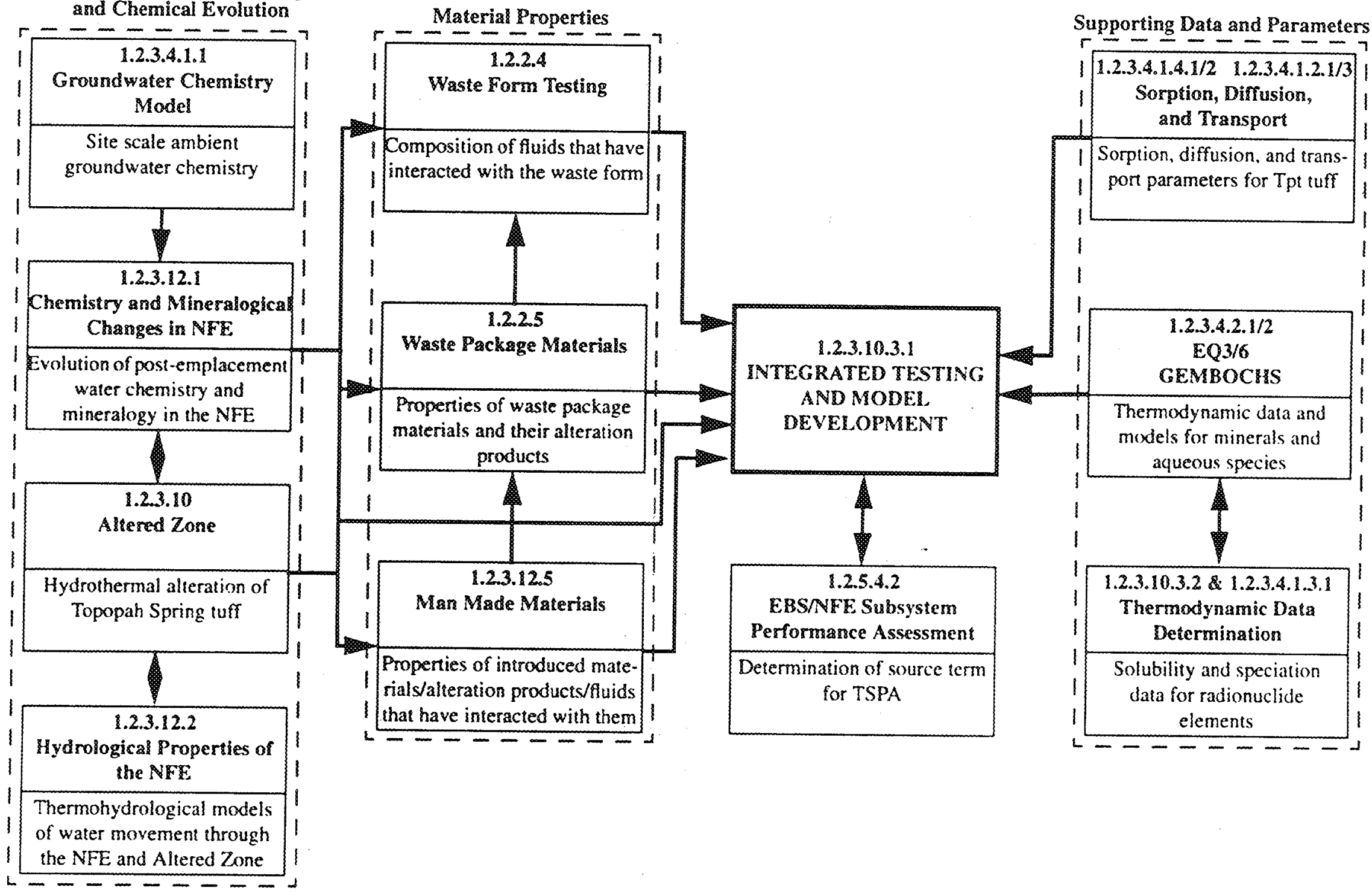

Figure 2. Information flow and relationship of Integrated Testing to other tasks. 


\subsubsection{Activities That Define the Near Field Environment and Models of Chemical, Mineralogical, and Hydrological Evolution in the Post Emplacement Period}

\subsubsection{Interface with Groundwater Chemistry Model Activities (WBS 1.2.3.4.1.1)}

There will be no direct interaction with this activity. The information provided by this activity to the NFE Geochemistry activity will help place bounds on the ambient water chemistry that is likely to contact and interact with the various components of the EBS/NFE. These bounds will provide guidance on the range in groundwater compositions to be used in initial experiments in the Integrated Testing task.

\subsubsection{Interface with NFE Geochemistry Activities (WBS 1.2.3.12.1)}

These activities will provide the chemical, mineralogical, and thermochemical scenarios that will define the evolution of the NFE over time. The input from these activities to Integrated Testing will be primarily via those activities that define the material properties of the EBS/NFE. These latter activities will utilize the information provided by NFE Geochemistry to assess the impact of NFE processes on material properties and their alteration products. Fluid compositional bounds that are defined by this activity will also be utilized to define the compositions to be used in Integrated Testing experiments and models.

\subsubsection{Interface with Altered Zone activities (WBS 1.2.3.10)}

These activities together with NFE Hydrology activities, will provide the coupled thermohydrological chemical models (reactive transport models) that will be used by Integrated Testing to predict the transport of radionuclides through the various components of the EBS/NFE. In addition, these activities coupled with NFE Geochemistry activities will help define the chemical and mineralogical properties of the NFE host rock that has been altered by hydrothermal reactions. A portion of this altered zone may extend to the edge of the EBS system and may display different radionuclide transport properties than the ambient host rock.

\subsubsection{Interface with Hydrological Properties of the NFE (WBS 1.2.3.12.2)}

Interaction with this activity will be indirect via the NFE Geochemistry activity. However, thermohydrological models coupled with chemical models (reactive transport models) that are developed by this activity and/or the Altered Zone activity will be utilized for modeling radionuclide transport and for interpreting transport experiments.

\subsubsection{Activisies That Define Material Properties of Flow-Path Components and Fluids}

\subsubsection{Interface with Waste Form Testing (WBS 1.2.2.4)}

The primary source of information regarding the composition of the radionuclide-bearing fluids that result from the interaction of the waste form and water in the NFE will come from the Waste Form Testing and Modeling activity. This information will be used to bound the compositions of 
fluids used in Activities G-20-3 and G-20-5 within the Integrated Testing task (Table 2). The Waste Form Testing activity will define the concentration and species of dissolved and colloidal radionuclides, and the $\mathrm{pH}$ and major element chemistry of fluids that are released from the waste form.

\subsubsection{Interface with Waste Package Materials activities (WBS 1.2.2.5)}

The first material a radionuclide-bearing solution derived from the waste form will contact is likely to be container materials or their alteration products. The activities in this WBS element will provide information regarding the nature of the barrier materials, their properties, and the corrosion or alteration products derived from them. This information will be used to define the flowpath components in the vicinity of the waste form to be used in Integrated Testing experiments and models. For some of these materials, transport parameters may also be provided by these activities (e.g, diffusion coefficients for radionuclides in non-metallic barriers). These activities will also provide information regarding possible packing, backfill, and invert components that might surround the waste package. These materials, if used, would likely be the second type of material contacted by a migrating radionuclide plume.

\subsubsection{Interface with NFE Man Made Materials activities (WBS 1.2.3.12.5)}

After traversing the EBS, a radionuclide-bearing solution may contact a number of materials or their alteration products that were used in the construction of the repository. The interface with the Man Made Materials activity primarily involves obtaining information regarding the material properties of components of the NFE that may comprise the flow path for radionuclides leaving the EBS, or that may affect the transport of radionuclides in the EBS. This task will provide mineralogical and physical property information for materials in the post-emplacement environment, as well as provide bounding compositions for fluids that have interacted with these materials. The nature and quantity of colloids that are derived from introduced materials will be bounded by this activity. The biological component of the NFE, and its effect on water chemistry, will also be defined by the Man Made Materials activity. The interaction of the surrounding host rock with introduced materials and/or the fluids derived from them may significantly affect the chemical and physical properties of the rock. This activity, together with the Altered Zone activity and the NFE Geochemistry activity, will define the properties of the host rock immediately adjacent to the EBS through which radionuclides must pass on exiting the EBS/NFE. Hence, the Man Made Materials activity will define the chemical and physical nature of various porous media and fluids that will comprise a significant component of the flow path of radionuclides through the EBS/NFE.

\subsubsection{Activities Supplying Supporting Data for Radionuclide Sorption, Transport, and Speciation Modeling}

In order to design appropriate experiments, interpret experimental results, and model the transport of radionuclides through the EBS/NFE, sorption, diffusion, transport, and thermodynamic data for radionuclides are necessary. 


\subsubsection{Interface with Dynamic Transport, Sorption and Diffusion Activities (WBS}

$1.2 .3 .4 .1 .4 .1 / 2 \& 1.2 .3 .4 .1 .2 .1 / 3)$

These activities have measured and/or compiled an extensive data base of parameters related to sorption, diffusive transport, and dynamic (advective/dispersive) transport of radionuclides. Sorption data include batch Kd's measured using crushed rock from Yucca Mountain formations as well as sorption model parameters measured using single minerals. Transport data include diffusive transport through intact tuff samples, and advective transport through crushed tuff, intact tuff, and fractured tuff samples. Most of this data have been collected at ambient temperatures. These data provide a starting point for similar data required for EBS/NFE materials at elevated temperatures. For that part of the EBS/NFE that includes tuff (e.g., crushed tuff backfill or altered tuff adjacent to the EBS), the data collected from this activity are especially relevant.

\subsubsection{Interface with EQ3/6/GEMBOCHS Activities (WBS 1.2.3.4.2.1/2)}

These activities interface with Integrated Testing by providing solubility and aqueous speciation data and models. This information is necessary to design appropriate dynamic and diffusive transport experiments, for interpreting the results of such experiments, and for predictive modeling.

\subsubsection{Interface with Thermodynamic Data Activities (WBS 1.2.3.10.3.2 \&1.2.3.4.1.3.1)}

The interface with these activities is indirect and exists via interaction with the EQ3/6/GEMBOCHS activity described above.

\subsubsection{Interface with EBS/NFE Subsystem Performance Assessment (WBS 1.2.5.4.2)}

The information inputs described above, together with the experimental and modeling results generated by the Integrated Testing activity will be used to place bounds on the concentrations of radionuclides likely to reach the outer boundary of the EBS/NFE. These bounding estimates will be provided to the EBS/NFE Subsystem Performance Assessment activity. Models developed in the Integrated Testing task will be abstracted for use in EBS/NFE PA. Subsystem performance assessment calculations will be used to predict the radionuclide source term to be used by TSPA. Subsystem PA calculations will also be used by Integrated Testing to identify important scenarios and potential EBS materials interactions that need to be addressed by the experimental and modeling aspects of the task.

\subsection{Quality Assurance}

The activities that have been performed under the original SIP and those to be started under this SIP are subject to procedure 033-YMP-QP2.8, "Quality Assurance Grading". Integrated Testing Activities (described below) G-20-2, G-20-3, G-20-5, G-20-6 have been graded and two (G-20-2, G-20-3) have recently been revised. With the exception of Integrated Testing Model Validation Activity (G-20-7), work performed under the experimental activities in Integrated Testing will 
initially be undertaken under non-Quality Affecting (QA) quality controls. It is expected that as the repository design becomes finalized and the types and properties of the actual materials making up the EBS/NFE flow paths are defined, the experimental activities that were initiated under non-QA controls will be regraded to Quality Affecting status. The need for preparation of Activity Plans, a more detailed planning document than the SIP, will be decided at the time of quality assurance grading or revision. Activity Plans will detail the specifics of how each Quality Affecting activity or activities will be carried out and will include technical details as well as schedules, planned training and records, use of Technical Implementing Procedures (TIPs), and expected results. TIPs may be needed for certain repetitive laboratory analyses. These will be prepared as necessary. All experimental and modeling activities in Integrated Testing will make use of controlled Scientific Notebooks, as indicated in procedure 033-YMP-QP 3.4. 


\subsection{SUMMARY OF PLANNED ACTIVITIES}

The activities comprising the Integrated Testing task (Table 2) may be divided into three focus areas:

1. Experimental measurement and compilation of parameters needed to model radionuclide transport through components of the EBS/NFE (Activities G-20-8 and G-20-2)

2. Experiments to measure the transport of radionuclides via advective/dispersive flow through components of the EBS/NFE (Activities G-20-8, G-20-3, and G-20-5)

3. The application, development, and validation of mechanistic, empiric, and abstracted models to make bounding predictions of radionuclide transport through the EBS/NFE (Activities G20-6 and G-20-7)

Table 2. Activities for Integrated Testing

\begin{tabular}{|l|l|}
\hline Activity & \multicolumn{1}{|c|}{ Title } \\
\hline G-20-2 & Transport model parameter experiments necessary to define the source term \\
\hline G-20-3 & Radionuclide transport experiments necessary to define the source term \\
\hline G-20-5 & Interactions of materials at repository conditions \\
\hline G-20-6 & Source term prediction and model development \\
\hline G-20-7 & Model validation \\
\hline G-20-8 & Planning/data assessment \\
\hline
\end{tabular}

\subsection{Experimental Measurement and Compilation of Model Parameters}

In order to predict transport of radionuclides using reactive transport models, a varicty of chemical data are necessary. These data may include: sorption parameters (Kd's, sorption isotherms, surface complexation constants, ion exchange constants, sorption kinetics); diffusion coefficients (for the species and media involved); solution speciation (aqueous complexes, redox state, colloids); solubility (considering both equilibrium and kinetic control); and, for some species, radioactive decay constants. The activities within this Integrated Testing focus area include experimental and data compilation tasks limited to the acquisition of sorption and diffusion parameters for selected radionuclides and selected EBS/NFE components. The other types of data required for modeling transport will be obtained from other YMP activities (Figure 2). 


\subsubsection{Planning/data assessment (Activity G-20-8)}

This activity will compile and assess existing sorption and diffusion data, identify data to be measured, and prioritize the experimental program. This activity will serve as the focal point for compilation of data provided through the information feeds identified in Figure 2.

\subsubsection{Transport model parameter experiments necessary to define the source term (Activity G-20-2)}

\subsubsection{Sorption}

As identified in Activity G-20-8, sorption measurements will be made for materials, radionuclides, and/or temperatures for which sorption data do not exist or are not known with confidence. Sorption measurements may include batch Kd measurements and measurement of surface complexation and ion exchange constants for whole materials or single minerals from EBS/NFE flowpath components.

\subsubsection{Diffusion}

Diffusion experiments will be undertaken to measure those parameters identified in Activity G20-8 that are needed to model the transport of radionuclides through heterogeneous fractured components of the EBS/NFE and through unconsolidated materials with a dual porosity distribution (e.g., coarsely crushed tuff).

\subsection{Experiments to Measure the Transport of Radionuclides}

\subsubsection{Planning/data assessment (Activity G-20-8)}

This activity will compile, assess, and identify data needs and define experimental approaches. Experimental test and strategy plans for transport experiments utilizing individual flow-path components (Activity G-20-3) and multiple flow-path components (G-20-5) will be prepared under this activity.

\subsubsection{Radionuclide transport experiments necessary to define the source term (Activity G-20-} 3)

Transport experiments will be undertaken for selected radionuclides or their analogues for which sorption and diffusion data are available or have been measured. Experiments for EBS/NFE flowpath components will be conducted at temperatures from ambient to boiling using solutions containing radionuclides at concentrations below their solubility limits. Major element concentrations 
and $\mathrm{pH}$ of these solutions will bound those expected for the various EBS/NFE flow-path components.

\subsubsection{Interactions of materials at repository conditions (Activity G-20-5)}

Under certain conditions, the interaction between two or more materials in the EBS/NFE may af fect the transport of radionuclides. For example, "contrast" in the physical and/or chemical properties for adjacent repository flow-path components may strongly influence the transport of radionuclides across their interface. Transport measurements using individual components of the EBS/NFE may not, therefore, capture the complexity of the transport process. Because of the proximity of the EBS/NFE fow-path components to the thermal source, temperature gradients may be large enough to significantly affect sorption, diffusion, and solubility limits within a single EBS/NFE flow-path component. Experiments to address these more complex situations will be addressed in this activity.

2.3 The Application, Development, and Validation of Models

\subsubsection{Source term prediction and model development (Activity G-20-6)}

Modeling activities will be undertaken at several levels of detail. Process oriented mechanistic models that account for fluid dynamics, radionuclide sorption and diffusion, radionuclide decay, and, where necessary, radionuclide precipitation/dissolution, will be used to interpret experimental results from transport experiments and to predict the transport of radionuclides through individual components of the EBS/NFE under various temperature and hydrologic conditions. These models will be used to compute bounding concentrations for radionuclides at various points within the EBS/NFE system for various hydrologic and thermal scenarios. Due to the complexity of these models, their incorporation into EBS/NFE subsystem performance assessment calculations will not be feasible. Development of look-up tables and/or simple functional relationships among important transport parameters, EBS/NFE material properties, hydrologic environment, and temperature will be developed that maintain the validity of the more complex models with respect to bounding estimates for radionuclide transport. It is these models that will be passed on to EBS/ NFE subsystem performance assessment.

\subsubsection{Model validation (Activity G-20-7)}

The bounding estimates of radionuclide flux from the EBS/NFE system should be known with a high degree of confidence. Hence, the models that will be used to make these bounding estimates and the parameters that the models employ need to be "validated", or tested sufficiently to build confidence in their results. The process by which confidence in the models will be gained constitutes the validation activities. The approach to validating the models will range from testing models against experimental data (bench scale, block scale, and drift scale), and against field 
observations of natural and perturbed systems. Bench scale experiments will be undertaken within the Integrated Testing task for validation activities. Block scale experiments will be undertaken in conjunction with the NFE Geochemistry and Altered Zone activities. Drift scale experiments for the EBS/NFE system are not planned for the Integrated Testing activity. These experiments, if undertaken, would be expected to cut across a number of activities. Where possible, field data from other activities and/or data published from other projects, such as DOE International Program collaborations, will be used for building confidence in the models. 


\subsection{Transport Model Parameter Experiments Necessary to Define the Source Term (Activity G-20-2)}

This activity encompasses experiments which determine sorption and diffusion parameters necessary for interpretation of radionuclide transport experiments (Activity G-20-3) and for modeling and predicting transport through the EBS/NFE (Activity G-20-6).

\subsubsection{Sorption Experiments}

Because flow-path components and fluids of the post-emplacement EBS/NFE differ significantly in their chemical and physical properties from Yucca Mountain rocks and present day groundwaters, their sorptive properties and capacities will differ significantly from those measured in the Dynamic Transpon, Sorption and Diffusion activities (WBS 1.2.3.4.1.4.1/2 \& 1.2.3.4.1.2.1/3) that have focused on the ambient conditions of the Yucca Mountain site (see Sec. 1.3.3.1). Sorption by EBS/NFE flow-path components may, depending on the materials used, provide a significant sorptive/solubility barrier to the release of radionuclides to the far field. For elements such as $\mathrm{U}, \mathrm{Np}$, and $\mathrm{Se}$, that are not strongly sorbed by the rocks at Yucca Mountain (3), EBS/NFE materials may significantly increase the overall retardation of these elements.

The interfaces and information flow identified in section 1.3 will be utilized to select and prioritize the materials to be used in the sorption experiments. Changes in the design for the EBS/NFE may require changes in the materials tested or the priority of the testing. The types of materials to be tested will include waste package filler, waste package corrosion products, hydrothermally altered backfill, cementitious materials, and altered Topopah Spring tuff. The physical, chemical, and mineralogical characteristics of these materials will be defined by the specific activities identified in section 1.3.

The radionuclide elements that will be addressed in these experiments will be selected based on priorities developed from previous studies of sorption and transport $(3,8)$, information from the Waste Form Testing area (WBS 1.2.2.4), and EBS/NFE PA calculations. A concurrent Integrated Testing activity, Planning/Data Assessment (Activity G-20-8) will compile and evaluate existing data and identify and prioritize the data that are needed.

Sorption experiments will be carried out using trace quantities (i.e., below solubility limit) of the relevant radionuclide or its analogue dissolved in a background electrolyte solution. The composition and $\mathrm{pH}$ of the radionuclide-bearing solutions will be formulated to maintain the radionuclide as a particular aqueous species and to maintain the porous media in a specific chemical state. Some solutions used in the experiments may be prepared to match the composition of fluids that are predicted to evolve in EBS/NFE materials (e.g., high pH fluids from concretes or relatively high ionic strength fluids that result when groundwater redissolves salts deposited during the repository dry-out period as a result of the post-emplacement thermal pulse). 
Sorption parameters will be determined at ambient to temperatures above $100^{\circ} \mathrm{C}$ using a method appropriate for the parameter of interest:

- batch equilibration measurements of partition coefficients and sorption isotherms for crushed material and/or unconsolidated flow-path components and fluids with compositions approximating and encompassing those that are expected in the EBS/NFE

- measurements of binary cation exchange isotherms using crushed material and/or single minerals to obtain cation exchange equilibrium constants.

- surface acidity titrations and sorption vs. pH measurements for determining surface complexation model parameters for single mineral phases and simple electrolyte solutions.

Sorption experiments will be performed using appropriate vessels that may include pressurized bombs and/or autoclaves for measurements above $100^{\circ} \mathrm{C}$. Standard chemical and instrumental techniques (counting, spectroscopic, titration) will be used for analyzing fluids and solid phases used in the experiments.

Partition coefficients (Kd's), sorption isotherms, and sorption model parameters, together with diffusion data, will be input to reactive transport models such as NUFT (5), that will be used to interpret transport experiments (Activity G-20-3) and to predict transport of radionuclides through the EBS/NFE (Activity G-20-6).

\subsubsection{Diffusion experiments}

Diffusion is expected to play an important role in mass transport of radionuclide species through EBS/NFE flow path components. Diffusion may control the rate of attainment of sorptive equilibrium (e.g., in zeolites and/or low porosity matrix materials) (6) and it may retard non-sorbing or weakly sorbing species during transport through fractured or other dual porosity media (4). In regions where advective/dispersive flow is zero or small, diffusion will dominate the transport of radionuclide species. Diffusion may thus control the movement of radionuclides to loci where advective/dispersive flow dominates.

The effective or simple diffusion coefficient defines the rate of diffusive flux of a non-reacting (conservative) species through a porous medium. This parameter is a function of the physical state of the medium (porosity, pore size distribution, tortuosity), the volumetric water content or saturation state of the medium, and the temperature. The apparent diffusion coefficient defines the rate of flux of a sorbing or reactive species through a porous medium in the absence of advective/ dispersive transport. Experiments using non-reactive tracers can be used to estimate effective diffusion coefficients for reactive species. Alternatively, diffusion of reactive species through a medium under conditions of no retardation (e.g., when the sorptive capacity of the medium is reached) can be used to directly determine the effective diffusion coefficient for the reactive species. Both apparent and effective diffusion coefficients will be measured and/or estimated in this activity.

The interfaces and information flow identified in section 1.3 will be utilized to select and prioritize the matcrials to be used in the diffusion experiments. Changes in the design for the EBS/NFE 
may require changes in the materials tested or the priority of the testing. The types of materials considered may range from homogeneous unconsolidated media (e.g., corrosion products) to consolidated fractured media (altered Topopah Spring tuff and altered concrete). The physical, chemical, and mineralogical characteristics of these materials will be defined by the specific activities identified in section 1.3.

The radionuclide elements that will be addressed in these experiments will be selected based on priorities developed from previous studies of sorption and transport $(3,8)$, information from the Waste Form Testing area (WBS 1.2.2.4), and EBS/NFE PA calculations. A concurrent Integrated Testing activity, Planning/Data Assessment (Activity G-20-8) will compile and evaluate existing data and identify and prioritize the data that are needed.

Diffusion experiments will be carried out using trace quantities of the relevant radionuclide or its analogue dissolved in a background electrolyte solution. The composition and $\mathrm{pH}$ of the radionuclide-bearing solutions will be formulated to maintain the radionuclide as a particular aqueous species, to maintain the porous media in a specific physical and chemical state, and to fix boundary conditions. To examine the effect of colloids on diffusion, the diffusing radionuclide species will be maintained in colloidal form for selected experiments.

Some solutions used in the experiments may be formulated to match the composition of fluids that are predicted to evolve in EBS/NFE materials. Other experiments may utilize solutions obtained from waste form leaching experiments. These solutions would be expected to contain multiple dissolved radionuclide species as well as colloids.

Diffusion will be measured for both saturated and unsaturated conditions at temperatures from ambient to near $100^{\circ} \mathrm{C}$. Experiments may include:

- measuring the rate of decrease in concentration of a radionuclide-traced solution in contact with a consolidated porous medium of specific geometry (i.e., wafers or cups of the medium)

- measuring the concentration profile of diffusing species in a porous medium or single crystal in contact with a radionuclide-traced solution for a specified period of time

- measuring the concentration of a diffusing species in a porous medium in contact with a radionuclide-traced medium at a specific volumetric saturation state

- measuring the electrical conductivity of a porous medium at a specific volumetric water content

Concentrations of radionuclides in the fluid phase will be measured by standard counting or spectroscopic techniques. Concentrations of radionuclides that have diffused into porous media will be measured by incrementally sampling the media (sectioning or grinding), dissolution or extraction of the samples, and counting. For single crystals, secondary ion mass spectrometry will be used to measure concentrations of the radionuclide versus position.

The rate of decrease of radionuclides with time in the contacting solution, or the concentration of radionuclide versus distance in the solid will be fit by either numerical or analytical methods to an appropriate solution to the diffusion equation for the relevant boundary conditions to determine either effective or apparent diffusion coefficients. 
The data collected from this activity will be used as input for reactive transport models, such as NUFT (5), that will be used for predicting transport through the EBS/NFE (Activity G-20-6).

3.2 Radionuclide Transport Experiments Necessary to Define the Source Term (Activity Gn 20-3)

In theory, if the chemical and physical properties of a fluid/porous medium are known, mechanistic reactive transport models could be used to predict the movement of radionuclides through the EBS/NFE. In practice, transport experiments are required to test and refine the models and to bound the actual radionuclide transport. This activity, together with Integrated Testing activity G20-5, will provide the experimental measurement of radionuclide transport through different EBS/ $\mathrm{NFE} /$ fluid/radionuclide systems under varying hydrologic and temperature regimes.

The interfaces and information flow identified in section 1.3 will be utilized to select and prioritize the materials to be used in the transport experiments. The EBS/NFE flow-path components used in the experiments will include consolidated materials such as altered concrete and Topopah Spring tuff, unconsolidated materials such as crushed tuff backfill and/or invert, and waste package container corrosion products and/or filling. Changes in the design for the EBS/NFE may require changes in the materials tested or the priority of the testing. The physical, chemical, and mineralogical characteristics of these materials will be defined by the specific activities identified in section 1.3.

The fluids that are used in the experiments will include simple analogues of those expected in the EBS/NFE, as well as fluids with compositions approximating those that have been identified in the activities that feed into Integrated Testing (Figure 2).

The radionuclide elements that will be addressed in these experiments will be selected based on priorities developed from previous studies of sorption and transport $(3,8)$, information from the Waste Form Testing area, and EBS/NFE PA calculations. A concurrent Integrated Testing activity, Planning/Data Assessment (Activity G-20-8) will compile and evaluate existing data and identify and prioritize the data that are needed.

Most experiments will be carried out under isothermal (from ambient temperature to near 100 ${ }^{\circ} \mathrm{C}$ ), steady state flow conditions using fully saturated and partially saturated materials. Some experiments will also be undertaken under conditions of infiltration or imbibition of fluids into initially unsaturated materials.

The experimental techniques and apparatuses may include:

- Column experiments for saturated and unsaturated transport through unconsolidated materials

- Core-flow experiments for saturated and unsaturated transport through consolidated intact and/or fractured materials 
- Centrifuge experiments for saturated and unsaturated transport through unconsolidated and consolidated intact and/or fractured materials (2)

- Core-flow and column experiments for infiltration and imbibition experiments

The experimental apparatuses will be designed to prevent flow around the edges of the materials, to maintain steady state flow conditions, to maintain isothermal conditions, and to monitor the hydrologic and chemical properties of media and the fluids emanating from them. Details of the experiments will be provided in documents prepared in Integrated Testing activity G-20-8.

The data from these experiments together with diffusion and sorption data will be used to bound the transport of radionuclides through specific components of the EBS/NFE. These experiments will provide a well constrained test of the reactive transport models that will be used to predict radionuclide transport in EBS/NFE components. However, because these will be bench scale experiments, the results may not be directly applicable to block and repository scales. For those EBS/ NFE materials with physical and/or chemical heterogeneities that are large compared to the scale of column and core-flow experiments, experiments at the block scale may also be undertaken in conjunction with the Altered Zone (WBS 1.2.3.10). NFE Geochemistry (WBS 1.2.3.12.1) and/or NFE Man Made Materials (WBS 1.2.3.12.5) activities. Plans for these experiments, if necessary, will be formulated under Integrated Testing activity $\mathrm{G}-20-8$.

\subsection{Interaction of Materials at Repository Conditions (Activity G-20-5)}

Although the scale of chemical and/or physical heterogeneities of individual EBS/NFE materials are smaller than those displayed in the host rock at the repository scale, heterogeneities introduced at the junction between material types and by thermal and hydrologic gradients may affect the transport of radionuclides. Because these types of heterogeneities cannot be addressed by isothermal transport experiments involving individual flow-path components, this activity will address them.

Integrated Testing activity G-20-8 together with information from EBS/NFE PA and NFE Geochemistry (WBS 1.2.3.12.1) and Man Made Materials (WBS 1.2.3.12.5) will be used to identify those interactions of materials (or heterogeneities) that may affect radionuclide transport through the EBS/NFE.

The materials used in these experiments will be similar to those used in Integrated Testing activity G-20-3. These experiments will be designed so that two or three flow-path components are assembled in the same apparatus. Because some of the interface effects may arise from materials interactions at elevated temperature, the flow-path components may need to be assembled and then hydrothermally treated prior to undertaking the transport experiment. Examples of assemblages with interfaces that display high chemical and physical "contrast" that might be used for these types of experiments include:

altered backfill $\rightarrow$ altered concrete $\rightarrow$ altered Topopah Spring tuff 
waste package corrosion products $\rightarrow$ altered backfill $\rightarrow$ altered concrete

where the arrows represent the direction of fluid flow and radionuclide transport. The results of these transport experiments, when compared to experiments that utilize individual flow-path components, will provide information to assess the importance of these types of heterogeneities on radionuclide transport.

In addition to material heterogeneities, steep thermal and/or hydrologic gradients may develop in EBS/NFE materials for extended periods. Information developed in the NFE Hydrology (WBS 1.2.3.12.2), NFE Geochemistry (WBS 1.2.3.12.1) and Man Made Materials (WBS 1.2.3.12.5) activities will be used to assess the extent and importance of these gradients. The feasibility of performing radionuclide transport experiments under thermal and hydrologic gradients will be addressed in Integrated Testing activity G-20-8.

The specifics of experimental design for this activity will be defined in Integrated Testing activity G-20-8, and will be closely coordinated with the NFE Geochemistry (WBS 1.2.3.12.1) and Man Made Materials (WBS 1.2.3.12.5) activities. It is expected that the experimental apparatuses and techniques will be similar to those developed and used in Integrated Testing activity G-20-3.

The information from this activity will be used to bound radionuclide transport through the EBS/ NFE, to refine and test reactive transport models, and to identify materials interactions that are important to radionuclide transport.

\subsection{Source Term Prediction and Model Development (Activity G-20-6)}

This activity is concerned with predicting the transport of radionuclides through the EBS/NFE and/or flow-path components and with testing and refining models to make these predictions. Modeling activities will be undertaken at several levels of detail. Because the knowledge base for specific materials, radionuclides, and sorption models differs, the level of detail brought to bear may vary for different radionuclides and/or flow-path components, and/or hydrologic scenarios.

Process oriented mechanistic models will be used wherever possible to interpret transport experiments and predict radionuclide transport. Reactive transport codes such as NUFT (5), FEHMN (11), and IDREACT (7), are numerical representations of mechanistic physical and chemical models. These codes vary in their ability to model specific chemical and physical process. It is expected that continued development of the capabilities of these codes will be necessary before the full range of solution phase, solid phase, and surface phase chemical processes describe radionuclide interaction with a porous medium can be modeled. Reactive transport codes that are able to model the full range of chemical and hydrologic processes affecting radionuclide transport can be used to make predictions beyond the temporal and physical/chemical conditions under which the model parameters were collected. These codes will be used to predict bounding concentrations for radionuclides at various points within the EBS/NFE system for various hydrologic and thermal scenarios. 
Refinements to these models (codes), other than boundary conditions, model parameters, etc. will be made by either the Altered Zone (WBS 1.2.3.10) or the NFE Hydrology (WBS 1.2.3.12.2) activities, if necessary. Due to the complexity of these models, they cannot be incorporated into EBS/NFE PA models without simplification. Process oriented models, provided they are sufficiently tested (Integrated Testing activity $\mathrm{G}-20-7$ ), can be used to bound the expected flux of radionuclides through the EBS/NFE for specific physical, chemical, and hydrological conditions.

Given bounding estimates of the flux of radionuclides under specific conditions, simplified models that relate radionuclide transport to EBS/NFE material property and state variables can be developed. These simplified models will be developed as single or multivariate functional relationships, or as look-up tables. The goal of the model abstraction process will be to maintain the validity of the more complex models with respect to bounding estimates for radionuclide transport, and at the same time, simplify the models so that PA calculations are feasible. Unlike process oriented mechanistic models, the simplified models cannot be used to predict transport for conditions outside those for which the model was developed. The abstracted models will be used by EBS/NFE PA for source term calculations.

\subsection{Source Term Model Validation (Activity G-20-7)}

The mechanistic and abstracted models that are applied and developed in Integrated Testing activ ity $\mathrm{G}-20-6$ will not be able to be fully validated in the sense that model predictions will perfectly match experimental outcomes. Even if model predictions and experimental observations agree for experiments carried out over relatively short time scales, the model may not necessarily be valid for longer time scales. Validating models utilized in the Integrated Testing and EBS/NFE PA tasks requires demonstrating that model predictions bound with a high degree of confidence the expected flux of radionuclides out of the EBS/NFE, i.e., the source term.

Although process oriented mechanistic models are complex and require a relatively large input of parameters, they have the best chance at representing reality because they represent physically realistic processes. The major effort of this task will be to test these models, and the submodels within them (e.g., sorption, diffusion, fluid flow), against as many different experimental observations at as many physical scales as possible. Wherever possible, model testing will be done for well-constrained laboratory and field experiments and/or observations.

Experiments that are specifically designed to test and validate the models will be performed in this activity. However, experiments performed in Integrated Testing activities G-20-3 or G-20-5 may, under appropriate controls, also be used for validation purposes. Experiments that are used to derive transport parameters and/or otherwise refine the mechanistic models will not be used for validation tests. Bench scale validation tests will range from single material isothermal steady siate flow tests, to multicomponent, transient transport tests. Sorption and diffusion tests may also be undertaken.

In addition to experiments undertaken in Integrated Testing, additional tests of the mechanistic models will be provided by results of block scale and drift scale tests undertaken in conjunction 
with other EBS/NFE activities. Similarly, where appropriate, observations from natural analogue and field studies, that are not Integrated Testing activities will also be used for model testing.

Abstracted models, because they are by necessity empiric, cannot be validated for temporal and physical/chemical conditions outside of those for which they were developed. Hence, validation of these models will focus on assuring that they will predict transport within the bounds estimated by experimental observations and mechanistic modeling for the range of conditions under which they were developed.

\subsection{Data Assessment/Planning (Activity G -20-8)}

This activity will compile, assess, and prioritize the experimental data collection activities in Integrated Testing activities G-20-2, G-20-3, and G-20-5. This activity will also define experimental testing approaches and strategies for Integrated Testing áctivities G-20-3, G-20-5, and G-20-7. Finally this activity will develop test and activity plans as required for specific quality affecting activities.

Although the schedule and milestones for Integrated Testing cannot be predicted with confidence; the following data compilation and planning documents were identified within this activity in the most recent plan for Integrated Testing:

- Report on Strategies of Integrated Testing

- Plans for Transport Studies in Unsaturated Materials

- Near Field Environment/Engineered Barrier System Transport Parameter Requirements

- Source Term Model Validation Strategy Plan

- Materials Interaction Experiments Plan 


\subsection{APPLICATION OF RESULTS}

The information provided by this investigation will bound the flux of radionuclides released from the EBS/NFE. This information will be used by the EBS/NFE PA task to model the behavior of the ensemble of waste packages under repository conditions and to estimate the source term to be used in TSPA. The information obtained in this investigation directly addresses the following issue and information needs (IN) taken from the SCP:

Issue 1.5: Will the waste package and repository engineered barriers meet the performance objective for radionuclide release as required by 10 CFR 60.113 ?

IN 1.5.3 Scenarios and models needed to predict the rate of radionuclide release from the waste package and engineered barrier system.

IN 1.5.5 Determination of the amount of radionuclides leaving the near-field environment of the waste package.

Through input to the above information needs, the results of the Integrated Testing activities will provide data to help resolve Issues 1.1, 1.5, and 1.9. 


\subsection{SCHEDULE AND MILESTONES}

A sequencing of the approximate start and completion times for the major activities discussed in this SIP is given in Table 3. The time scale shown is relative because the exact completion dates for the various activities depends on the budget and progress made by other tasks in the YMP. As a general guide, contingent on budget and progress, a time scale of about 5 years is envisioned for the content of this SIP. The current YMP network charts should be consulted for more details and the disposition of milestones for the different activities.

Table 3. Schedule for Integrated Testing activities

\begin{tabular}{|l|c|}
\hline \multicolumn{1}{|c|}{ Activity } & Time (relative) \\
\hline $\begin{array}{l}\text { G-20-2 } \\
\text { Model parameters }\end{array}$ & \\
\hline $\begin{array}{l}\text { G-20-3 } \\
\text { Transport experiments }\end{array}$ & \\
\hline $\begin{array}{l}\text { G-20-5 } \\
\text { Material interactions }\end{array}$ & \\
\hline $\begin{array}{l}\text { G-20-6 } \\
\text { Source term model }\end{array}$ & \\
\hline $\begin{array}{l}\text { G-20-7 } \\
\text { Model validation }\end{array}$ & \\
\hline $\begin{array}{l}\text { G-20-8 } \\
\text { Data assessment/planning }\end{array}$ & \\
\hline
\end{tabular}


1. Buscheck, T.A., and Nitao, J.J. 1993. Repository-heat-driven hydrothermal flow at Yucca Mountain, Part I: Modeling and analysis. Nuclear Tech. 104:418

2. Conca, J. 1992. Transport in unsaturated flow systems using centrifuge techniques. In Proceedings of the DOE/Yucca Mountain Site Characterization Project Radionuclide Adsorption Workshop at Los Alamos National Laboratory, September 11-12, 1990. LA-12325-C. Los Alamos National Laboratory, Los Alamos, NM.

3. Meijer, A. 1992. A strategy for the derivation and use of sorption coefficients in performance assessment calculations for the Yucca Mountain site. In Proceedings of the DOE/Yucca Mountain Site Characterization Project Radionuclide Adsorption Workshop at Los Alamos National Laboratory, September 11-12, 1990. LA-12325-C. Los Alamos National Laboratory, Los Alamos, NM.

4. Neretnieks, I. 1980. Diffusion in rock matrix: An important factor in radionuclide migration? J. Geophys. Res. 85:4379

5. Nitao, J. 1995. Reference Manual for the NUFT Flow and Transport Code. Lawrence Livermore National Laboratory report UCRL-ID 113520. Livermore, CA.

6. Rundberg, R.S. 1987. Assessment Report on the Kinetics of Radionuclide Adsorption on Yucca Mountain Tuff. Los Alamos National Laboratory report LA-11026-MS. Los Alamos, NM.

7. Steefel, C.I., and Lasaga, A.C. 1994. A coupled model for transport of multiple chemical species and kinetic precipitation/dissolution reactions with application to reactive flow in single phase hydrothermal systems. Amer. J. Sci. $294: 529$

8. Triay, I.R., Mitchell, A.J., and Ott, M.A. 1992. Radionuclide migration laboratory studies for validation of batch-sorption data. In Proceedings of the DOE/Yucca Mountain Site Characterization Project Radionuclide Adsorption Workshop at Los Alamos National Laboratory, September 11-12, 1990. LA-12325-C. Los Alamos National Laboratory, Los Alamos NM.

9. US DOE. 1988. Site Characterization Plan: Yucca Mountain Site, Nevada Research and Development Area. DOE/RW-0199. Washington DC

10. YMP. 1987. Scientific Investigation Plan for Integrated Testing. SIP-4. LLNL-YMP

11.Zyvoloski, G.A., Robinson, B.A., Dash, Z.V., and Trease, L.L. 1995. Users Manual for the FEHMN Application. LA-UR-94-3788, Rev. 1. Los Alamos National Laboratory, Los Alamos, NM. 